\title{
Asymptotic stability and transient optimality of economic MPC without terminal conditions
}

\author{
Lars Grüne $^{1}$, Marleen Stieler ${ }^{2}$ \\ Mathematical Institute, University of Bayreuth, 95440 Bayreuth, Germany
}

\begin{abstract}
We consider an economic nonlinear model predictive control scheme without terminal constraints or costs. We give conditions based on dissipativity and controllability properties under which the closed loop is practically asymptotically stable. Under the same conditions we prove approximate transient optimality of the closed loop on finite time intervals. Two numerical examples illustrate our theoretical findings.
\end{abstract}

Keywords: economic MPC, practical asymptotic stability, transient performance, controllability, stabilizability

\section{Introduction}

Economic Model Predictive Control (MPC) has attracted considerable attention during the last couple of years. Due to the availability of fast and reliable solution algorithms for the underlying optimal control problems and an increasing demand for efficiency, e.g., in terms of consumptions of resources and energy or regarding a reduction of the environmental impact, the idea to use more sophisticated "economic" objectives directly in an MPC formulation is both natural and appealing. The hope is that by solving an optimal control

Email addresses: lars.gruene@uni-bayreuth.de (Lars Grüne), marleen.stieler@uni-bayreuth.de (Marleen Stieler)

${ }^{1}$ Supported by the European Union under the 7th Framework Programme FP7- PEOPLE2010-ITN, Grant agreement number 264735-SADCO

${ }^{2}$ Supported by the International Doctorate Program "Identification, Optimization and Control with Applications in Modern Technologies" within the Elite Network of Bavaria 
problem in each sampling instant, the resulting closed loop will also be optimal with respect to the chosen economic criterion, at least in an approximate sense. Results which show that this is indeed the case were given, e.g., in [1, 3] in an averaged infinite horizon sense. Besides optimality, stability of the closed loop with respect to a given optimal equilibrium is often of interest. Stability results for economic MPC schemes can be found, e.g., in [3, 7, 10].

In all of the references just cited, terminal conditions - i.e., terminal constraints and/or costs - or other modifications of the "plain" finite horizon economic optimal control problem are used in order to ensure stability and performance estimates. While such mechanisms are able to improve the performance of MPC schemes, they are often avoided in practice. Reasons for this are that terminal constraints limit the operating region of the controller and may pose problems in numerically solving the optimal control problem in each step of the MPC scheme. Terminal costs, on the other hand, may be complicated to design particularly in time variant settings. Moreover, although terminal costs may in principle be used without terminal constraints, they typically provide only a local approximation to the true cost-to-go and thus require terminal constraints in order to ensure that the optimized trajectories end up in a region where the terminal cost attains meaningful values. Finally, and most importantly, stability like behaviour and good performance are often observed without any terminal conditions. Thus, the purpose of this paper is to explain why and under which conditions this is the case.

Like in many of the references, above, in this paper we assume a strict dissipativity condition which in particular implies the existence of an optimal steady state $x^{e}$, cf. [11]. For this setting, it is already known that - under appropriate conditions, for details see [8] - Economic MPC without terminal constraints yields closed loop trajectories which are approximately optimal in an averaged infinite horizon sense. Moreover, under an exponential turnpike assumption, cf. [5, 12, the trajectories converge to a neighborhood of $x^{e}$ and there exists at least one time horizon for which the closed loop trajectory is also approximately optimal in a finite horizon sense. Since (approximate) optimality 
in an infinite horizon averaged sense is in fact a rather weak optimality concept (as the trajectory may be far from optimal on any finite time interval) the latter is important because it tells us that the closed loop trajectory when initialized away form the optimal steady state approaches this equilibrium in an approximately optimal way. In other words, the closed loop is not only optimal on average in the long run but also shows near optimal performance during its transient phase.

The present paper builds upon the results of 8$]$ and improves them in several directions. First of all, due to a refined error analysis we will be able to remove the exponential turnpike property from the list of assumptions. Although numerical results indicate that exponential turnpike is a widely spread property, this nevertheless simplifies the assumptions and extends the applicability of our results. Second, we will prove practical asymptotic stability (instead of mere convergence as in [8]) and provide a corresponding practical Lyapunov function. The particular form of this function will then enable us to prove near optimal transient performance for arbitrary finite time intervals (instead of for only a single one as in [8). Last but not least, we present our results under less technical assumptions. This is achieved by restricting ourselves to two particular and in a sense opposing - settings: on the one hand, we consider fully nonlinear systems with compact state constraints sets which are locally controllable around $x^{e}$. For this setting we present sufficient conditions for practical asymptotic stability and approximately optimal transient performance. On the other hand, we consider strictly convex affine-linear-quadratic problems without any state constraints for which we show that practical asymptotic stability of the MPC closed loop holds if and only if the system is stabilizable. While there are certainly various intermediate settings which are of interest, we consider these two cases as prototypical and hope that their treatment enables the interested reader to carry over our results to his or her favourite setting.

The remainder of this paper is organized as follows. In Section 2 we define our notation and formulate the underlying optimal control problem. We introduce the concept of model predictive control and give a sufficient condition 
for practical asymptotic stability, namely the existence of a practical Lyapunov function. In Section 3 two settings of optimal control problems are given for which we derive the existence of a practical Lyapunov function. By using this Lyapunov function, approximate optimal behaviour of the closed loop during the transient phase is proven in Section 4. In Section 5 we present two examples with numerical tests that illustrate the theoretical results of Section 3 and 4 . Section 6 gives an conclusion of the paper and an outlook to future research. Since the proofs of our main theorems are quite technical they are moved to the separate Section 7.

\section{Problem formulation and preliminary results}

We consider nonlinear discrete time control systems given by

$$
x(k+1)=f(x(k), u(k))
$$

for some $f: X \times U \rightarrow X$, with $X$ and $U$ normed spaces that denote the state space and the control space. The solution of system (1) for a control sequence $u=(u(0), u(1), \ldots, u(K-1)) \in U^{K}$ emanating from the initial value $x$ is denoted by $x_{u}(k, x), k=0, \ldots, K-1$. The sets $\mathbb{X}$ and $\mathbb{U}$ denote the admissible states and controls. For a given initial value $x \in \mathbb{X}$, a control sequence $u \in \mathbb{U}^{K}$ is called admissible if $x_{u}(k, x) \in \mathbb{X}$ holds for all time instants $k=0, \ldots, K$. The set of all admissible control sequences is denoted by $\mathbb{U}^{K}(x)$. For the infinite case $u=(u(0), u(1), \ldots) \in U^{\infty}$ we define the sets $\mathbb{U}^{\infty}$ and $\mathbb{U}^{\infty}(x)$ similarly.

For a given stage cost $\ell: X \times U \rightarrow \mathbb{R}$ we define the finite horizon cost functional

$$
J_{N}(x, u):=\sum_{k=0}^{N-1} \ell\left(x_{u}(k, x), u(k)\right),
$$

and the corresponding optimal value function

$$
V_{N}(x):=\inf _{u \in \mathbb{U}^{N}(x)} J_{N}(x, u) .
$$

In the sequel we assume that for all $x \in \mathbb{X}$ and all $N \in \mathbb{N}$ there is a control sequence $u_{N, x}^{\star} \in \mathbb{U}^{N}(x)$, such that the equality $V_{N}(x)=J_{N}\left(x, u_{N, x}^{\star}\right)$ holds, i.e. 
$u_{N, x}^{\star}$ solves the optimal control problem of minimizing $J_{N}(x, u)$ with respect to $u \in \mathbb{U}^{N}(x)$. This particularly includes the assumption $\mathbb{U}^{N}(x) \neq \emptyset$ for all $x \in \mathbb{X}$ which holds if and only if $\mathbb{X}$ is a viable set. Note that we do not require uniqueness of the optimal control sequences. In case of non-uniqueness, $u_{N, x}^{\star}$ denotes one of the minimizing control sequences.

The optimal control problem just defined can be used in order to define a feedback law using the following model predictive control (MPC) iteration. Fixing an optimization horizon $N \in \mathbb{N}$, at each time instant $n$ we perform the following steps:

1. Measure the current state $x=x(n)$ of the system.

2. Solve the optimization problem of minimizing $J_{N}(x, u)$ with respect to $u \in \mathbb{U}^{N}(x)$ subject to $x_{u}(0, x)=x$ and $x_{u}(k+1, x)=f\left(x_{u}(k, x), u(k)\right)$. Denote the resulting optimal control sequence by $u_{N, x}^{\star}$.

3. Apply the first element of $u_{N, x}^{\star}$ as a feedback control value until the next time instant, i.e., define the feedback law $\mu_{N}(x):=u_{N, x}^{\star}(0)$.

The resulting $M P C$ closed loop system is given by $x(n+1)=f\left(x(n), \mu_{N}(x(n))\right)$. Trajectories of this system with initial value $x \in \mathbb{X}$ will be denoted by $x_{\mu_{N}}(n, x)$

As the MPC feedback law is derived from minimizing (2), questions about the optimality properties of the closed loop naturally arise. Here, we will investigate the values

$$
J_{K}^{c l}\left(x, \mu_{N}\right):=\sum_{n=0}^{K-1} \ell\left(x_{\mu_{N}}(n, x), \mu_{N}\left(x_{\mu_{N}}(n, x)\right)\right),
$$

for arbitrary $K \in \mathbb{N}$. Moreover, stability properties of the closed loop are of interest and - as we will see - form an important prerequisite for approximate optimality estimates. Both issues have been addressed in 8 which forms the basis for this paper. However, instead of stability only convergence was established, see [8, Theorems 7.1 and 7.6] and approximate optimality could only be established for certain $K \in \mathbb{N}$ but not for arbitrary $K$, cf. [ 8 , Remark 7.7]. Moreover, these properties could only be proved under the condition that certain error terms converge sufficiently fast as $N \rightarrow \infty$, cf. the discussion after [8, 
Theorem 7.1]. All these limitations will be overcome in this paper. Another contribution is the observation that essentially the same Lyapunov function which can be used in economic MPC with terminal conditions [ 6 , 3, can also be used in our setting without terminal constraints.

For the definition of stability we will make use of the following classes of comparison functions

$$
\begin{aligned}
\mathcal{L} & :=\left\{\delta: \mathbb{R}_{0}^{+} \rightarrow \mathbb{R}_{0}^{+} \mid \delta \text { continuous and decreasing with } \lim _{k \rightarrow \infty} \delta(k)=0\right\}, \\
\mathcal{K} & :=\left\{\alpha: \mathbb{R}_{0}^{+} \rightarrow \mathbb{R}_{0}^{+} \mid \alpha \text { continuous, strictly increasing with } \alpha(0)=0\right\}, \\
\mathcal{K}_{\infty} & :=\{\alpha \in \mathcal{K} \mid \alpha \text { unbounded }\}, \\
\mathcal{K} \mathcal{L} & :=\left\{\beta: \mathbb{R}_{0}^{+} \times \mathbb{R}_{0}^{+} \rightarrow \mathbb{R}_{0}^{+} \mid \beta \text { continuous, } \beta(\cdot, t) \in \mathcal{K}, \beta(r, \cdot) \in \mathcal{L}\right\},
\end{aligned}
$$

Stability will be considered for optimal steady states defined as follows.

Definition 2.1. A pair $\left(x^{e}, u^{e}\right) \in \mathbb{X} \times \mathbb{U}$ that satisfies the condition $f\left(x^{e}, u^{e}\right)=$ $x^{e}$ is called steady state or equilibrium for the control system (1). A steady state is optimal, if it solves the optimization problem

$$
\min _{x \in \mathbb{X}, u \in \mathbb{U}} \ell(x, u) \text { s.t. } f(x, u)-x=0 .
$$

Definition 2.2. Let $x^{e} \in \mathbb{X}$ be an equilibrium for the closed loop system, i.e. $x^{e}=f\left(x^{e}, \mu\left(x^{e}\right)\right)$. The equilibrium is called practically asymptotically stable w.r.t. $\varepsilon \geq 0$ on a set $S \subseteq \mathbb{X}$ with $x^{e} \in S$ if there exists $\beta \in \mathcal{K} \mathcal{L}$ such that

$$
\left\|x_{\mu}(k, x)-x^{e}\right\| \leq \max \left\{\beta\left(\left\|x-x^{e}\right\|, k\right), \varepsilon\right\}
$$

holds for all $x \in S$ and all $k \in \mathbb{N}$. The equilibrium is globally practically asymptotically stable w.r.t. $\varepsilon \geq 0$ if (5) holds on $S=\mathbb{X}$.

A sufficient condition for this stability property is the existence of a practical Lyapunov function in the following sense.

Definition 2.3. A function $V: \mathbb{X} \rightarrow \mathbb{R}$ is a practical Lyapunov function w.r.t. $\delta>0$ for the closed loop system on a set $S \subseteq \mathbb{X}$ with $x^{e} \in S$, if there are 
$\alpha_{1}, \alpha_{2} \in \mathcal{K}_{\infty}$ and $\alpha_{3} \in \mathcal{K}$ such that

$$
\alpha_{1}\left(\left\|x-x^{e}\right\|\right) \leq V(x) \leq \alpha_{2}\left(\left\|x-x^{e}\right\|\right)
$$

holds for all $x \in \mathbb{X}$ and

$$
V(f(x, \mu(x))) \leq V(x)-\alpha_{3}\left(\left\|x-x^{e}\right\|\right)+\delta
$$

holds for all $x \in S$.

The proof of the following theorem is standard but we provide some details for the convenience of the reader.

Theorem 2.4. Let $V$ be a practical Lyapunov function w.r.t. some $\delta>0$ on a set $S \subseteq \mathbb{X}$. Assume that either $S=\mathbb{X}$ or $S=V^{-1}[0, L]:=\{x \in \mathbb{X} \mid V(x) \leq L\}$ for some $L>\alpha_{2}\left(\alpha_{3}^{-1}(\delta)\right)+\delta$. Then $x^{e}$ is practically asymptotically stable on $S$ w.r.t. $\varepsilon=\alpha_{1}^{-1}\left(\alpha_{2}\left(\alpha_{3}^{-1}(\delta)\right)+\delta\right)$.

Proof: Inequality (7) and the assumption on $S$ implies $f(x, \mu(x)) \in S$ for all $x \in S$, i.e., forward invariance of $S$. Define $\eta:=\alpha_{2}\left(\alpha_{3}^{-1}(\delta)\right)+\delta$ and $P:=$ $V^{-1}[0, \eta]$. We claim that $P$ is also forward invariant. To this end, we pick $x \in P$, i.e., $V(x) \leq \eta$, and distinguish two cases:

Case 1: $\alpha_{3}\left(\left\|x-x^{e}\right\|\right) \geq \delta$. In this case we get

$$
V\left(f(x, \mu(x)) \leq V(x)-\alpha_{3}\left(\left\|x-x^{e}\right\|\right)+\delta \leq V(x)-\delta+\delta=V(x) \leq \eta\right.
$$

implying $f(x, \mu(x)) \in P$.

Case 2: $\alpha_{3}\left(\left\|x-x^{e}\right\|\right)<\delta$. In this case we get $\left\|x-x^{e}\right\|<\alpha_{3}^{-1}(\delta)$, implying $V(x)<\alpha_{2}\left(\alpha_{3}^{-1}(\delta)\right)$ and thus

$$
V\left(f(x, \mu(x)) \leq V(x)-\alpha_{3}\left(\left\|x-x^{e}\right\|\right)+\delta<\alpha_{2}\left(\alpha_{3}^{-1}(\delta)\right)+\delta=\eta\right.
$$

which again yields $f(x, \mu(x)) \in P$.

Now by continuity there exists $c>1$ with $\alpha_{2}\left(\alpha_{3}^{-1}(c \delta)\right) \leq \eta$. For $x \in S \backslash P$ we have $V(x) \geq \eta$ and consequently $\alpha_{3}\left(\left\|x-x^{e}\right\|\right) \geq \alpha_{3}\left(\alpha_{2}^{-1}(V(x))\right) \geq \alpha_{3}\left(\alpha_{2}^{-1}(\eta)\right) \geq$ 
$c \delta$ for all $x \in S \backslash P$. This implies $\alpha_{3}\left(\left\|x-x^{e}\right\|\right)-\delta \geq(1-1 / c) \alpha_{3}\left(\left\|x-x^{e}\right\|\right)$ and thus

$$
V(f(x, \mu(x))) \leq V(x)-\left(1-\frac{1}{c}\right) \alpha_{3}\left(\left\|x-x^{e}\right\|\right)
$$

for all $x \in S \backslash P$. Hence, $V$ is a Lyapunov function on $S \backslash P$ in the sense of [9, Definition 2.18] and [9, Theorem 2.20] yields practical asymptotic stability w.r.t. the exceptional set $P$. Since $x \in P$ implies $V(x) \leq \eta$ and thus $\left\|x-x^{e}\right\| \leq$ $\alpha_{1}^{-1}(\eta)=\varepsilon$, this proves the assertion.

\section{Stability results}

In this section we formulate our main results on practical stability of the economic MPC closed loop system under two different sets of assumptions. The first applies to general nonlinear dynamics and costs. The respective assumptions read as follows.

Assumption 3.1 (Strict dissipativity). The optimal control problem of minimizing (2) is strictly dissipative, i.e., there is an equilibrium $\left(x^{e}, u^{e}\right) \in \mathbb{X} \times \mathbb{U}$, a function $\alpha_{\ell} \in \mathcal{K}_{\infty}$ and a storage function $\lambda: X \rightarrow \mathbb{R}$ such that

$$
\min _{u \in \mathbb{U}} \tilde{\ell}(x, u) \geq \alpha_{\ell}\left(\left\|x-x^{e}\right\|\right)
$$

holds for all $x \in \mathbb{X}$, where $\tilde{\ell}$ denotes the rotated stage costs

$$
\tilde{\ell}(x, u):=\ell(x, u)+\lambda(x)-\lambda(f(x, u))-\ell\left(x^{e}, u^{e}\right) .
$$

In the next assumptions we use the balls $\mathcal{B}_{\delta}\left(x^{e}\right):=\left\{x \in \mathbb{X} \mid\left\|x-x^{e}\right\|<\delta\right\}$ for $\delta>0$.

Assumption 3.2 (Continuity and compactness). The state and control constraint set $\mathbb{X}$ and $\mathbb{U}$ are compact, the functions $f, \ell$ and $\lambda$ are continuous, $\lambda$ is Lipschitz continuous on a ball $\mathcal{B}_{\delta}\left(x^{e}\right)$ around $x^{e}$ and $\tilde{\ell}$ satisfies the inequality

$$
\tilde{\ell}(x, u) \leq \alpha\left(\left\|x-x^{e}\right\|\right)+\alpha\left(\left\|u-u^{e}\right\|\right)
$$

for all $x \in \mathbb{X}, u \in \mathbb{U}$ and a suitable $\alpha \in \mathcal{K}_{\infty}$. 
We remark that under Assumption 3.1 the function $\tilde{\ell}$ is zero in $\left(x^{e}, u^{e}\right)$. Hence, in the finite dimensional case with $\mathbb{X} \subseteq \mathbb{R}^{n}$ and $\mathbb{U} \subseteq \mathbb{R}^{m}$ inequality follows from continuity of $\tilde{\ell}$.

Assumption 3.3 (Local controllability on $\mathcal{B}_{\varepsilon}\left(x^{e}\right)$ ). There is $\varepsilon>0, M^{\prime} \in$ $\mathbb{N}, C>0$ such that $\forall x \in \mathcal{B}_{\varepsilon}\left(x^{e}\right) \exists u_{1} \in \mathbb{U}^{M^{\prime}}(x), u_{2} \in \mathbb{U}^{M^{\prime}}\left(x^{e}\right)$ with

$$
x_{u_{1}}\left(M^{\prime}, x\right)=x^{e}, x_{u_{2}}\left(M^{\prime}, x^{e}\right)=x
$$

and

$$
\begin{array}{r}
\max \left\{\left\|x_{u_{1}}(k, x)-x^{e}\right\|,\left\|x_{u_{2}}\left(k, x^{e}\right)-x^{e}\right\|,\left\|u_{1}(k)-u^{e}\right\|,\right. \\
\left.\left\|u_{2}(k)-u^{e}\right\|\right\} \leq C\left\|x-x^{e}\right\|
\end{array}
$$

for $k=0,1, \cdots, M^{\prime}-1$.

Assumption 3.4 (Finite time controllability into $\mathcal{B}_{\varepsilon}\left(x^{e}\right)$ ). For $\varepsilon>0$ from Assumption 3.3 there is $K \in \mathbb{N}$ such that for each $x \in \mathbb{X}$ there is $k \leq K$ and $u \in \mathbb{U}^{k}(x)$ with

$$
x_{u}(k, x) \in \mathcal{B}_{\varepsilon}\left(x^{e}\right)
$$

Assumption 3.5 (Polynomial bounds). There are constants $C_{1}, C_{2}, p, \eta>$ 0 such that

$$
C_{1}\left(\left\|x-x^{e}\right\|^{p}\right) \leq \tilde{\ell}(x, u) \leq C_{2}\left(\left\|x-x^{e}\right\|^{p}+\left\|u-u^{e}\right\|^{p}\right)
$$

holds for all $x \in \mathcal{B}_{\eta}\left(x^{e}\right), u \in \mathcal{B}_{\eta}\left(u^{e}\right)$ with $x^{e}, u^{e}$ and $\tilde{\ell}$ from Assumption 3.1.

In order to formulate our first main stability theorem, we need the following additional definition.

Definition 3.6. For the rotated stage cost $\tilde{\ell}$ from Assumption 3.1, we define $\widetilde{J}_{N}(x, u)$ and $\widetilde{V}_{N}(x)$ similar to (2) and (3) with $\tilde{\ell}$ in place of $\ell$. 
We remark that the optimal trajectories minimizing the original cost functional $J_{N}(x, u)$ are in general different from those minimizing $\widetilde{J}_{N}(x, u)$. Hence, MPC closed loop trajectories w.r.t. both stage costs are not expected to coincide, either. Indeed, we will see in Section 5 that they may differ considerably.

Theorem 3.7. Consider an economic MPC problem without terminal constraints satisfying Assumptions 3.1 3.4. Then there exists $N_{0} \in \mathbb{N}$ and functions $\delta \in \mathcal{L}$ and $\alpha_{V} \in \mathcal{K}_{\infty}$ such that the inequalities

$$
\alpha_{\ell}\left(\left\|x-x^{e}\right\|\right) \leq \widetilde{V}_{N}(x) \leq \alpha_{V}\left(\left\|x-x^{e}\right\|\right)
$$

and

$$
\widetilde{V}_{N}\left(f\left(x, \mu_{N}(x)\right)\right) \leq \widetilde{V}_{N}(x)-\alpha_{\ell}\left(\left\|x-x^{e}\right\|\right)+\delta(N)
$$

hold for all $N \geq N_{0}$ and $x \in \mathbb{X}$. In particular, the functions $\widetilde{V}_{N}$ are practical Lyapunov functions for the economic MPC closed loop system and the closed loop is practically asymptotically stable w.r.t. $\varepsilon \rightarrow 0$ as $N \rightarrow \infty$. If, moreover, Assumption 3.5 holds, then the function $\delta(N)$ converges to 0 exponentially fast as $N \rightarrow \infty$, i.e., there are $C>0$ and $\theta \in(0,1)$ with $\delta(N) \leq C \theta^{N}$.

The proof of this theorem can be found in Section 7 .

Remark 3.8. Note that our assumptions are not much more restrictive than those needed in [3] for proving stability for terminal constrained economic MPC. Strict dissipativity $3^{3}$ and continuity are also assumed in this reference, Assumption 3.3 is slightly stronger but conceptually similar to Assumption 2 in [3] and Assumption 3.4 will hold if we restrict $\mathbb{X}$ to the feasible set $\mathcal{X}_{N}$ from [3].

Our second set of assumption covers unconstrained linear quadratic problems. In this setting, we make the following assumptions.

\footnotetext{
${ }^{3}$ Strict dissipativity in 3 is defined by means of a merely positive definite function $\alpha_{\ell}$ while here we require $\alpha_{\ell}$ in Assumption 3.1 to be of class $\mathcal{K}_{\infty}$. However, since we assumed $\mathbb{X}$ to be compact, this does not make a difference.
} 
Assumption 3.9 (Linear quadratic problem). The dynamics and the cost functions are given by

$$
f(x, u)=A x+B u+c \quad \text { and } \quad \ell(x, u)=x^{T} R x+u^{T} Q u+s^{T} x+v^{T} u
$$

with $x \in \mathbb{R}^{n}, u \in \mathbb{R}^{m}, A, B, R, Q$ are matrices and $s, v$ are vectors of appropriate dimensions with $R$ and $Q$ symmetric and positive definite.

Assumption 3.10 (No constraints). There are no state and control constraints, i.e., $\mathbb{X}=\mathbb{R}^{n}$ and $\mathbb{U}=\mathbb{R}^{m}$.

Note that in this setting there exists a unique optimal steady state $x^{e}$ in the sense of Definition 2.1. Moreover, [5, Proposition 4.5] shows that $x^{e}$ is strictly dissipative with $\tilde{\ell}$ satisfying Assumption 3.5 .

Theorem 3.11. Consider an economic MPC problem without terminal constraints satisfying Assumptions 3.9 and 3.10 and let $x^{e}$ be the optimal steady state. Then $x^{e}$ is practically asymptotically stable on each compact subset $S \subset$ $\mathbb{R}^{n}$ w.r.t. $\varepsilon \rightarrow 0$ as $N \rightarrow \infty$ if and only if the pair $(A, B)$ is stabilizable.

In this case, the problem is strictly dissipative and the functions $\widetilde{V}_{N}$ are practical Lyapunov functions for the closed loop and $\varepsilon$ converges to 0 exponentially fast in $N$.

Again, the proof is found in Section 7

\section{Transient performance}

In this section we use the results from the last section in order to prove an approximate transient optimality property of economic MPC without terminal constraints. In order to formulate the concept of transient optimality, assume that the MPC closed loop is practically asymptotically stable, implying $x_{\mu_{N}}(K, x) \rightarrow x^{e}$ as $N \rightarrow \infty$ and $K \rightarrow \infty$. Then transient optimality means that among all trajectories $x_{u}(k, x)$ satisfying $\left\|x_{u}(K, x)-x^{e}\right\| \leq\left\|x_{\mu_{N}}(K, x)-x^{e}\right\|$, the MPC closed loop trajectories are those with the smallest cost $J_{K}(x, u)-$ up to an error term which vanishes as $N \rightarrow \infty$ and $\left\|x_{\mu_{N}}(K, x)-x^{e}\right\| \rightarrow 0$. 
We define

$$
\mathbb{U}_{\varepsilon}^{K}(x):=\left\{u \in \mathbb{U}^{K}(x) \mid x_{u}(K, x) \in \mathcal{B}_{\varepsilon}(x)\right\}
$$

Theorem 4.1. Assume that $x^{e}$ is practically asymptotically stable on a set $S \subseteq$ $\mathbb{X}$ w.r.t. $\varepsilon=\varepsilon(N)$ for the economic MPC closed loop with Lyapunov function $\widetilde{V}_{N}$ satisfying (12), (13). Assume that there exists $\alpha_{\lambda} \in \mathcal{K}_{\infty}$ with $|\lambda(x)| \leq \alpha_{\lambda}(\| x-$ $\left.x^{e} \|\right)$ for all $x \in \mathbb{X}$. Let $\varepsilon_{K, N}:=\left\|x_{\mu_{N}}(K, x)-x^{e}\right\| \leq \max \left\{\beta\left(\left\|x-x^{e}\right\|, K\right), \varepsilon(N)\right\}$.

Then the inequality

$$
J_{K}^{c l}\left(x, \mu_{N}(x)\right) \leq \inf _{u \in \mathbb{U}_{\varepsilon_{K, N}}^{K}(x)} J_{K}(x, u)+\alpha_{V}\left(\varepsilon_{K, N}\right)+2 \alpha_{\lambda}\left(\varepsilon_{K, N}\right)+K \delta(N)
$$

holds for all $K, N \in \mathbb{N}$ and all $x \in S$.

Proof: First, by induction from 13 we obtain

$$
\sum_{k=0}^{K-1} \tilde{\ell}\left(x_{\mu_{N}}(k, x), \mu_{N}\left(x_{\mu_{N}}(k, x)\right)\right) \leq \widetilde{V}_{N}(x)-\widetilde{V}_{N}\left(x_{\mu_{N}}(K)\right)+K \delta(N) .
$$

Second, from the dynamic programming principle

$$
\widetilde{V}_{N}(x)=\inf _{u \in U^{K}(x)}\left\{\widetilde{J}_{K}(x, u)+\widetilde{V}_{N-K}\left(x_{u}(K, x)\right)\right\}
$$

and 12 we obtain for all $K \in\{1, \ldots, N\}$ and $u \in \mathbb{U}_{\varepsilon}^{K}(x)$

$$
\begin{aligned}
\widetilde{J}_{K}(x, u) & =\underbrace{\widetilde{J}_{K}(x, u)+\widetilde{V}_{N-K}\left(x_{u}(K, x)\right)}_{\geq \widetilde{V}_{N}(x)}-\underbrace{\widetilde{V}_{N-K}\left(x_{u}(K, x)\right)}_{\leq \alpha_{V}(\varepsilon)} \\
& \geq \widetilde{V}_{N}(x)-\alpha_{V}(\varepsilon)
\end{aligned}
$$

and we note that for $K \geq N$ non-negativity of $\tilde{\ell}$ implies the inequality $\widetilde{J}_{K}(x, u) \geq$ $\widetilde{V}_{N}(x)$ for all $u \in \mathbb{U}^{K}(x)$, implying again 16 . Third, we have

$$
\sum_{k=0}^{K-1} \tilde{\ell}\left(x_{u}(k, x), u(k)\right)=\widetilde{J}_{K}(x, u)=\lambda(x)+J_{K}(x, u)-\lambda\left(x_{u}(K, x)\right)
$$


and $\widetilde{V}_{N}(x) \geq 0$. Using these inequalities for all $u \in \mathbb{U}_{\varepsilon_{K, N}}^{K}(x)$ we obtain

$$
\begin{aligned}
& J_{K}^{c l}\left(x, \mu_{N}(x)\right) \stackrel{177}{=} \sum_{k=0}^{K-1} \tilde{\ell}\left(x_{\mu_{N}}(k, x), \mu_{N}\left(x_{\mu_{N}}(k, x)\right)\right) \\
& -\lambda(x)+\lambda\left(x_{\mu_{N}}(K, x)\right) \\
& \leq{ }^{15} \leq \widetilde{V}_{N}(x)-\widetilde{V}\left(x_{\mu_{N}}(K, x)\right)+K \delta(N) \\
& -\lambda(x)+\lambda\left(x_{\mu_{N}}(K, x)\right) \\
& \stackrel{16]}{\leq} \widetilde{J}_{K}(x, u)+\alpha_{V}\left(\varepsilon_{K, N}\right)-\widetilde{V}\left(x_{\mu_{N}}(K, x)\right)+K \delta(N) \\
& -\lambda(x)+\lambda\left(x_{\mu_{N}}(K, x)\right) \\
& \stackrel{17}{=} J_{K}(x, u)+\alpha_{V}\left(\varepsilon_{K, N}\right)-\widetilde{V}\left(x_{\mu_{N}}(K, x)\right)+K \delta(N) \\
& -\lambda\left(x_{u}(K, x)\right)+\lambda\left(x_{\mu_{N}}(K, x)\right) \\
& \leq J_{K}(x, u)+\alpha_{V}\left(\varepsilon_{K, N}\right)+K \delta(N)+2 \alpha_{\lambda}\left(\varepsilon_{K, N}\right)
\end{aligned}
$$

implying the desired inequality.

Remark 4.2. $\quad$ i) Note that all assumptions of Theorem 4.1 are satisfied if either Assumptions 3.13 .4 or Assumptions 3.93 .10 are satisfied. In the latter case the existence of $\alpha_{\lambda}$ follows because in the linear quadratic setting $\lambda$ is either a linear or a quadratic function, cf. [5]. Moreover, if Assumption 3.5 holds then $\delta(N)$ converges to 0 exponentially fast as $N \rightarrow \infty$, implying that the error terms on the right hand side of (14) converge to 0 if $K, N \rightarrow \infty$ with $K \leq c N$ for some $c>0$. In addition, in this case $\tilde{\ell}$ and $\widetilde{V}$ have identical polynomial growth near $x^{e}$, implying that the convergences $\beta(r, k) \rightarrow 0$ as $k \rightarrow \infty$ and $\varepsilon(N) \rightarrow 0$ as $N \rightarrow \infty$ are exponentially fast and thus all error terms in (14) converge to 0 exponentially fast as $K, N \rightarrow \infty$ with $K \leq c N$ for some $c>0$.

ii) Optimal trajectories minimizing (2) in general do not end up near $x^{e}$, see, e.g., the examples in [5]. Hence, for $u \in \mathbb{U}^{K}(x)$ the value $J_{K}(x, u)$ can be much smaller than $J_{K}^{c l}\left(x, \mu_{N}\right)$ and thus estimate (14) can only hold if we restrict the control sequences to $u \in \mathbb{U}_{\varepsilon_{K, N}}^{K}(x)$. In words, the estimate 
states that among all trajectories converging to a neighborhood of $x^{e}$, the ones generated by MPC are - up to the error terms - the ones with the lowest cost $J_{K}(x, u)$.

\section{Numerical example}

Example 5.1. Consider the one-dimensional economic growth model from [4] with dynamics

$$
x(k+1)=u(k)
$$

and stage cost

$$
\ell(x, u)=-\ln \left(A x^{\alpha}-u\right)
$$

with $A=5$ and $\alpha=0.34$. We impose state constraints $\mathbb{X}=[0,10]$ and control constraints $\mathbb{U}=[0.1,5]$. The optimal steady state of the control system is given by $\left(x^{e}, u^{e}\right)=\left(x^{e}, x^{e}\right)$ with $x^{e} \approx 2.23$ and related stage cost $\ell\left(x^{e}, u^{e}\right) \approx-1.467$. The problem is strictly dissipative with storage function $t^{4} \lambda(x)=\sigma x, \sigma=0.2306$. Figure 1 shows that the closed loop trajectories converge into a neighborhood of the optimal equilibrium, which is getting smaller as $N$ increases. As Figure 2 shows the neighborhood is shrinking exponentially fast. This confirms our theoretical results since all Assumptions of Theorem 3.7 are fulfilled by this example.

Next we illustrate the approximate optimal behaviour of the MPC closed loop during the transient phase. To this end, we compare the MPC controllers $\mu_{N}$ computed using four different cost functions:

\footnotetext{
${ }^{4}$ For linear control systems with strictly convex cost the storage function can always be chosen linear, see [6] or [5]. In this case, strict dissipativity translates to strong duality which can be used in order to compute the storage function $\lambda$. For linear systems with non-strictly convex cost functions, a constructive approach to compute $\lambda$ can be found in [5]. For nonlinear systems, computing $\lambda$ is in general a difficult task. We note, however, that the knowledge of $\lambda$ is not needed for implementing our economic MPC controller but only for its analysis.
} 


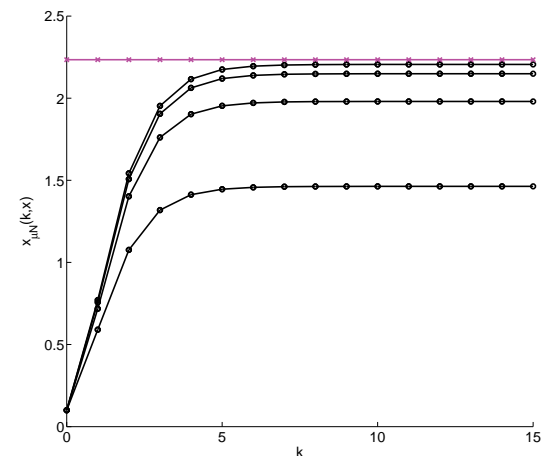

Figure 1: Closed loop for $N=2, \ldots, 5$ (bottom to top) and $x=0.1$.

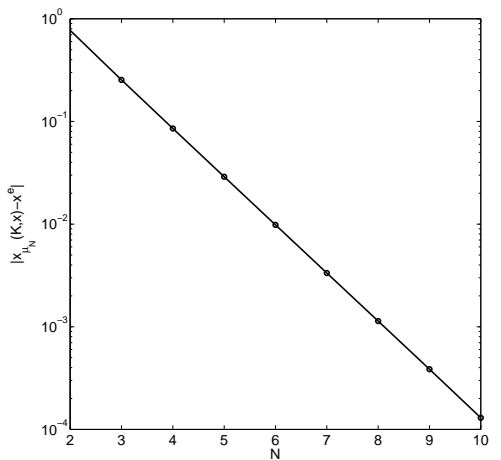

Figure 2: Distance from the closed loop to $x^{e}$ at final time for $N=2, \ldots, 10, x=0.1$.

- the original economic stage cost $\ell$

- the rotated stage cost $\tilde{\ell}$ from (9)

- the stabilizing quadratic stage cost $\ell^{\text {stab }}(x, u)=\left(x-x^{e}\right)^{2}+\left(u-u^{e}\right)^{2}$

$$
\rightsquigarrow \mu_{N}^{s t a b}
$$

- the stabilizing quadratic stage cost

$$
\begin{aligned}
\ell^{t a y l}(x, u)=\ell\left(x^{e}, u^{e}\right)+\frac{1}{2} 0.12125\left(x-x^{e}\right)^{2} & \\
& -0.05315\left(x-x^{e}\right)\left(u-u^{e}\right)+\frac{1}{2} 0.05315\left(u-u^{e}\right)^{2}
\end{aligned}
$$

whose weights were derived from a 2nd order Taylor approximation of $\ell$ in $\left(x^{e}, u^{e}\right)$

Figure 3 shows the closed loop trajectories for $\mu_{N} \in\left\{\mu_{N}^{e c o}, \mu_{N}^{r o t}, \mu_{N}^{\text {stab }}, \mu_{N}^{\text {tayl }}\right\}$ for $N=5$. One sees that the feedback $\mu_{N}^{\text {stab }}$ yields the fastest convergence towards $x^{e}$, followed by $\mu_{N}^{\text {tayl }}$ and $\mu_{N}^{\text {rot }}$. The trajectory for the original economic costs $\ell$ controlled by $\mu_{N}^{e c o}$ behaves similarly to the rotated problem but only converges to a neighborhood of $x^{e}$.

Now, in order to investigate approximate optimal transient performance, for 


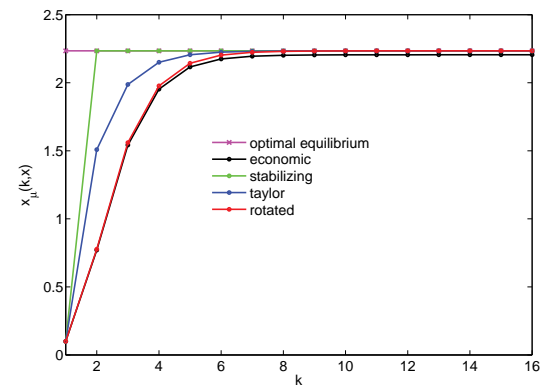

Figure 3: Closed loop trajectories for $N=$ $5, x=0.1$ with respect to different stage costs.

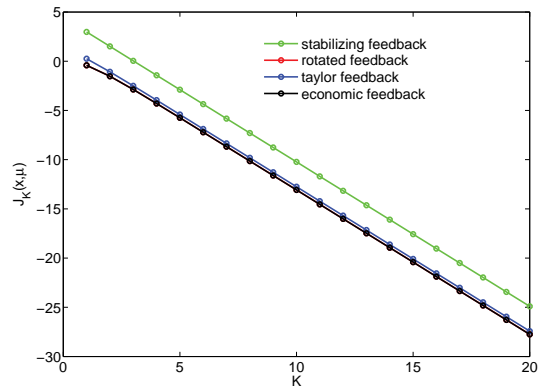

Figure 4: $J_{K}^{c l}\left(x, \mu_{N}\right)$ for $N=5, x=0.1$ and varying $K$ subject to different feedbacks $\mu_{N}$

given $N$ and $K$ we calculate $J_{K}^{c l}\left(x, \mu_{N}\right)$ for the different MPC controller 5 , In Figure 4 we show the values for fixed $N=5$ and varying $K=1, \ldots, 20$. One sees that the values of the cost functionals are almost parallel, which is due to the fact that the difference is mainly accumulated in the first few time steps. The value of $J_{K}^{c l}\left(x, \mu_{N}^{e c o}\right)$ is almost identical to $J_{K}^{c l}\left(x, \mu_{N}^{r o t}\right)$ and both are better than the other feedbacks. Observe that the merely practical stability of $\mu_{N}^{e c o}$ does not have a visible effect in this comparison.

Next we investigate two fixed values for $K$ and varying optimization horizons $N$ in Figures 5 and 6 . While in Figure $5 \mu_{n}^{\text {eco }}$ yields the best performance for all $N$, Figure 6 reveals that $J_{K}^{c l}\left(x, \mu_{N}^{e c o}\right)$ might not yield the best performance for very small $N$, but converges to $J_{K}^{c l}\left(x, \mu_{N}^{r o t}\right)$ as $N$ increases and is slightly better than $\mu_{N}^{r o t}$ and considerably better than $\mu_{N}^{\text {tayl }}$ and $\mu_{N}^{\text {stab }}$ for most values of $N$.

Example 5.2. The second example is a two-dimensional tank reactor model (Example 3.2 in [8]) with affine linear dynamics

$$
x(k+1)=\left(\begin{array}{cc}
0.8353 & 0 \\
0.1065 & 0.9418
\end{array}\right) x(k)+\left(\begin{array}{c}
0.00457 \\
-0.00457
\end{array}\right) u(k)+\left(\begin{array}{c}
0.5559 \\
0.5033
\end{array}\right)
$$

\footnotetext{
${ }^{5}$ In this comparison $J_{K}^{c l}\left(x, \mu_{N}\right)$ is always evaluated using the economic cost $\ell$. The different cost functions only refer to the computation of $\mu_{N}(x)$ in Step 2 of the MPC algorithm.
} 


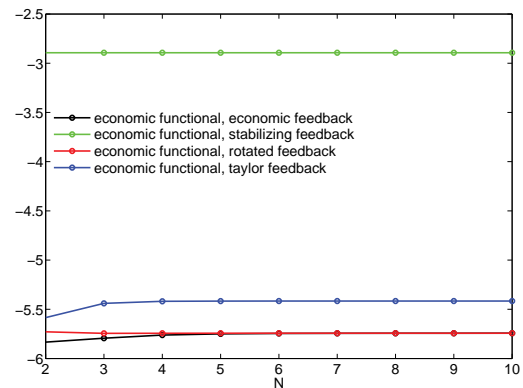

Figure 5: $J_{K}^{c l}\left(x, \mu_{N}\right)$ for $K=5, x=0.1$ and varying $N$ with different feedbacks $\mu_{N}$.

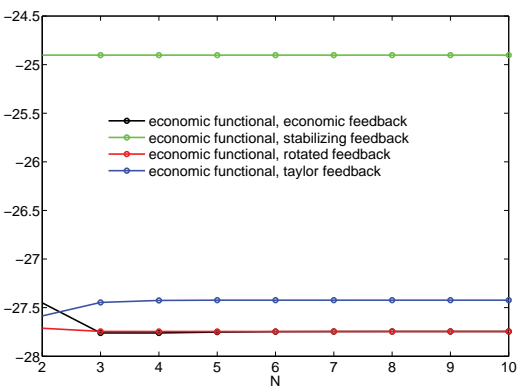

Figure $6: J_{K}^{c l}\left(x, \mu_{N}\right)$ for $K=20, x=0.1$ and varying $N$ with different feedbacks $\mu_{N}$.

and quadratic stage costs $\ell(x, u)=\|x\|^{2}+0.05 u^{2}$. State and control constraints are given by $\mathbb{X}=[-100,100]^{2}, \mathbb{U}=[-10,10]$. The optimal steady state of this problem is $x^{e} \approx(3.546,14.653)^{T}, u^{e} \approx 6.163$ with cost $\ell\left(x^{e}, u^{e}\right) \approx 229.1876$. As in the previous example, we observe that the closed loop trajectories converge into a neighborhood of $x^{e}$ which is shrinking as $N$ increases. This confirms the result in Theorem 3.11, since the pair $(A, B)$ in the dynamics is stabilizable and the stage costs are striclty convex.

The problem is strictly dissipative with respect to the storage function $\lambda(x)=$ $(-368.6684,-503.5415) x$, and the comparison of the closed loop trajectories subject to the original and the rotated stage costs in Figure 7 shows that the trajectory based on the rotated stage costs converges exactly to the optimal steady state. For this example we only compare $\mu_{N}^{e c o}$ and $\mu_{N}^{\text {rot }}$ since by [5, Proposition 4.5$]$ the rotated costs $\tilde{\ell}$ of this problem are quadratic, i.e., $\tilde{\ell}$ coincides with the "canonical" choice of stabilizing quadratic costs $\ell^{\text {stab }}$ and with its 2 nd order Taylor approximation $\ell^{\text {tayl }}$. Our simulations show that for fixed $N=10$ and varying $K=1, \ldots, 100$ the closed loop values for $\mu_{N}^{\text {eco }}$ and $\mu_{N}^{\text {rot }}$ are virtually indistinguishable, cf. Figure 8, For fixed $K$ and varying $N$, Figures 9 and 10 show (again) that even though the performance of $\mu_{N}^{e c o}$ might not be the best for small $N, J_{K}^{c l}\left(x, \mu_{N}^{e c o}\right)$ converges to $J_{K}^{c l}\left(x, \mu_{N}^{r o t}\right)$ as $N$ increases and $\mu_{N}^{\text {eco }}$ (at least slightly) outperforms $\mu_{N}^{\text {rot }}$ for sufficiently large $N$. 


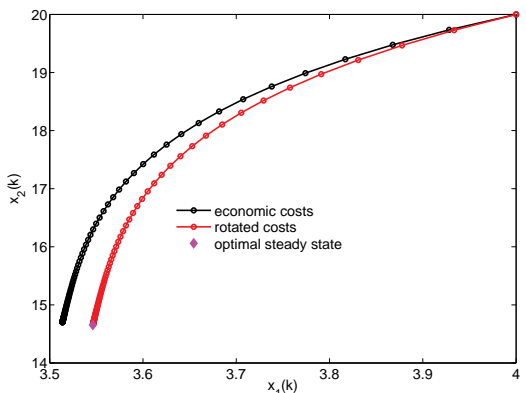

Figure 7: Closed loop trajectories subject to different feedbacks $\mu_{N}$ for $N=10, x=$ $(4,20)^{T}$ and optimal steady state (diamond).

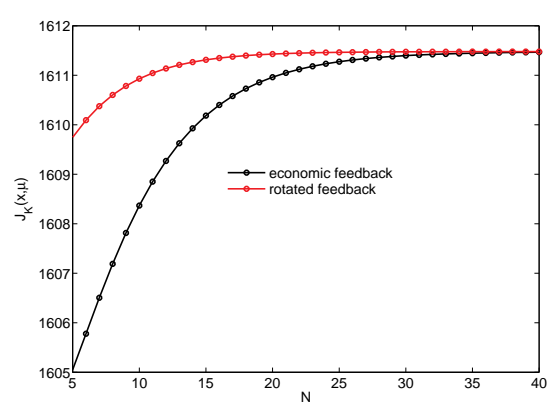

Figure 9: $J_{K}^{c l}\left(x, \mu_{N}\right)$ for $K=4, \quad x_{0}=$ $(4,20)^{T}$ and varying $N$ subject to different feedbacks $\mu_{N}$.

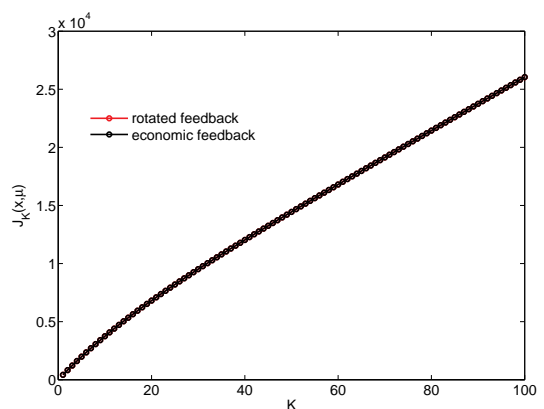

Figure 8: $J_{K}^{c l}\left(x, \mu_{N}\right)$ for $N=10, \quad x=$ $(4,20)^{T}$ and varying $K$ subject to different feedbacks $\mu_{N}$.

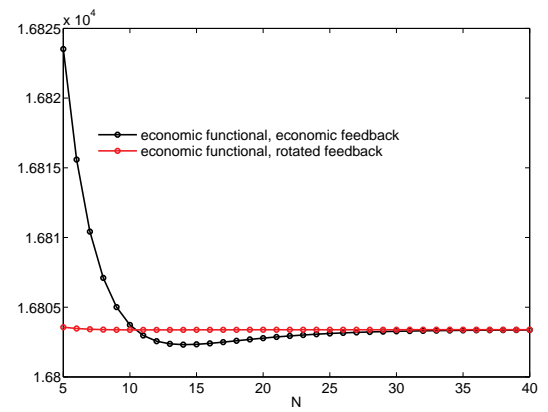

Figure 10: $J_{K}^{c l}\left(x, \mu_{N}\right)$ for $K=60, x_{0}=$ $(4,20)^{T}$ and varying $N$ subject to different feedbacks $\mu_{N}$.

\section{Conclusions}

We have presented conditions that yield a practical Lyapunov function and, hence, practical asymptotic stability for the MPC without terminal conditions. The conditions are given for two types of discrete time finite horizon optimal control problems. It was shown that a Lyapunov function for these control systems is given by the optimal value function with respect to the rotated stage costs. Compared to previous results for economic MPC with terminal conditions, our analysis shows that the price to pay for the simplifications gained from avoiding terminal conditions is that we only obtain practical instead of 
true asymptotic stability of the optimal steady state. However, our results also allow to conclude approximate transient optimality on arbitrary finite time intervals, a result which currently has no counterpart in economic MPC with terminal conditions.

The numerical simulations confirmed our theoretical results and have moreover shown that for sufficiently large $N$ economic MPC exhibits the best transient performance among the MPC variants we tested. Nevertheless, the simulations also revealed that MPC based on the rotated stage costs can be an interesting alternative, since in more involved problems it may be computationally infeasible to use these sufficiently large $N$. For small $N$, however, MPC based on the rotated cost can be advantageous since it is superior in terms of stability and the transient performance converges faster for increasing $N$, see, e.g., Figures 9 and 10, A rigorous investigation of this topic will be subject of future research, along with the attempt to compute quantitative estimates for the optimization horizon $N$ needed in order to ensure given error bounds. Moreover, we intend to investigate transient performance also for economic MPC schemes with terminal conditions.

\section{Proof of stability results}

Proof of Theorem 3.7: The proof of the theorem is split into three steps. In step one we show how to obtain inequality 12 , in step two we deal with inequality 13 . Finally, in step three the exponential convergence of $\delta$ in $(13)$ is deduced. In what follows we will make use of the results in [8]. We mention that the formulas taken from [8] look slightly different here since all the cost functionals in [8] are averaged, i.e., $J_{N}(x, u)$ is divided by $N$.

Step 1: proof of 12 . Strict dissipativity (Assumption 3.1 yields $\widetilde{V}_{N}(x) \geq$ $\alpha_{\ell}\left(\left\|x-x^{e}\right\|\right) \forall x \in \mathbb{X}$. The upper bound in 12 can be deduced from Assumptions $3.2-3.4$ as follows.

In case $x \notin \mathcal{B}_{\varepsilon}\left(x^{e}\right)$ with $\varepsilon$ from Assumptions 3.3 , 3.4, there is a control sequence $u$ that steers $x$ into the equilibrium in at most $M^{\prime}+K$ steps $\left(M^{\prime}, K\right.$ 
independent of $x$ ) and stays there for an arbitrary number of time steps. Therefore, for each $N \in \mathbb{N}$ it holds

$$
\widetilde{V}_{N}(x) \leq \widetilde{J}_{N}(x, u) \leq \widetilde{J}_{M^{\prime}+K}(x, u) \leq\left(M^{\prime}+K\right) \cdot \max _{x \in \mathbb{X}, u \in \mathbb{U}} \tilde{\ell}(x, u)=: \bar{C} .
$$

In case $x \in \mathcal{B}_{\varepsilon}\left(x^{e}\right)$, there is a control sequence $u \in U^{M^{\prime}}(x)$ with $x_{u_{1}}\left(M^{\prime}, x\right)=x^{e}$ and $\left\|x_{u_{1}}(k, x)-x^{e}\right\| \leq C\left\|x-x^{e}\right\|,\left\|u_{1}(k)-u^{e}\right\| \leq C\left\|x-x^{e}\right\|$ for all $k=$ $0, \ldots, M^{\prime}-1$. Together with 10$)$ this yields

$$
\begin{aligned}
\widetilde{V}_{N}(x) & \leq \widetilde{J}_{N}\left(x, u_{1}\right) \leq \widetilde{J}_{M^{\prime}}\left(x, u_{1}\right) \\
& \leq \sum_{k=0}^{M^{\prime}-1} \alpha\left(\left\|x_{u_{1}}(k, x)-x^{e}\right\|\right)+\alpha\left(\left\|u_{1}(k)-u^{e}\right\|\right) \\
& \leq 2 M^{\prime} \alpha\left(C\left\|x-x^{e}\right\|\right)=: \tilde{\alpha}\left(\left\|x-x^{e}\right\|\right) .
\end{aligned}
$$

Clearly, $\tilde{\alpha} \in \mathcal{K}_{\infty}$. If $\tilde{\alpha}\left(\left\|x-x^{e}\right\|\right) \geq \bar{C}$ for $x \notin \mathcal{B}_{\varepsilon}\left(x^{e}\right)$, we get $\widetilde{V}_{N}(x) \leq \tilde{\alpha}\left(\left\|x-x^{e}\right\|\right)$ for all $x \in \mathbb{X}$. Otherwise, we multply $\tilde{\alpha}\left(\left\|x-x^{e}\right\|\right)$ by a constant $\bar{K}$ such that $\bar{K} \tilde{\alpha}\left(\left\|x-x^{e}\right\|\right) \geq \bar{C}$ for $x \notin \mathcal{B}_{\varepsilon}\left(x^{e}\right)$. Combining these considerations yields

$$
\widetilde{V}_{N}(x) \leq \alpha_{V}\left(\left\|x-x^{e}\right\|\right) \quad \text { for } \quad \alpha_{V}(r):=\max \{1, \bar{K}\} \tilde{\alpha}(r)
$$

and, hence, 12 .

Step 2: proof of (13). Under the assumptions of [8, Theorem 7.6] (for details see below) the last formula in the proof of this theorem shows that there is $\tilde{\delta} \in \mathcal{L}$ such that for all $x \in \mathbb{X}, K \in \mathbb{N}$ and $N$ sufficiently large we get

$$
\widetilde{J}_{K}^{c l}\left(x, \mu_{N}(x)\right) \leq \widetilde{V}_{N}(x)-\widetilde{V}_{N}\left(x_{\mu_{N}}(K, x)\right)+\tilde{\delta}(N) .
$$

for a function $\tilde{\delta} \in \mathcal{L}$ (a precise upper bound for $\tilde{\delta}$ is given in Step 3 of this proof, below).

Now, inserting $K=1$ to 18 and observing $x_{\mu_{N}}(1, x)=f\left(x, \mu_{N}(x)\right)$ yields

$$
\begin{aligned}
\widetilde{V}_{N}\left(f\left(x, \mu_{N}(x)\right)\right) & \leq \widetilde{V}_{N}(x)-\tilde{\ell}\left(x, \mu_{N}(x)\right)+\tilde{\delta}(N) \\
& \leq \widetilde{V}_{N}(x)-\alpha_{\ell}\left(\left\|x-x^{e}\right\|\right)+\tilde{\delta}(N),
\end{aligned}
$$

which is equivalent to inequality 13 in Theorem 3.7 if we set $\delta(N)=\tilde{\delta}(N)$.

It remains to show that the assumptions of Theorem 3.7, i.e., Assumptions 3.13 .4 imply the relevant assumptions of [8, Theorem 7.6]. These are: 
1. Strict dissipativity and $\lambda$ bounded on $\mathbb{X}$.

2. (a) There is $C^{\prime} \geq 0$ such that $\forall x \in \mathbb{X}, \forall \varepsilon>0$ the quantity

$$
Q_{\varepsilon}:=\#\left\{k \in\{0, \ldots, N-1\}:\left\|x_{u_{N, x}^{\star}}(k, x)-x^{e}\right\| \leq \varepsilon\right\}
$$

satisfies $Q_{\varepsilon} \geq N-\frac{C^{\prime}}{\alpha_{\ell}(\varepsilon)}$, with $\alpha_{\ell}$ from Assumption 3.1 and $u_{N, x}^{\star}$ denoting the optimal control for $J_{N}(x, u)$.

(b) There is $\tilde{C}^{\prime} \geq 0$ such that $\forall x \in \mathbb{X}, \forall \varepsilon>0$ the quantity

$$
\tilde{Q}_{\varepsilon}:=\#\left\{k \in\{0, \ldots, N-1\}:\left\|x_{\tilde{u}_{N, x}^{\star}}(k, x)-x^{e}\right\| \leq \varepsilon\right\}
$$

satisfies $\tilde{Q}_{\varepsilon} \geq N-\frac{\tilde{C}^{\prime}}{\alpha_{\ell}(\varepsilon)}$, with $u_{N, x}^{\star}$ denoting the optimal control for $\widetilde{J}_{N}(x, u)$.

3. There are $\bar{\delta}>0, N_{0} \in \mathbb{N}, \gamma_{V} \in \mathcal{K}_{\infty}$ such that for all $\rho \in(0, \bar{\delta}]$, all $N \in$ $\mathbb{N}_{\geq N_{0}}$ and all $x \in \mathcal{B}_{\rho}\left(x^{e}\right)$ it holds

$$
\begin{aligned}
& \left|V_{N}(x)-V_{N}\left(x^{e}\right)\right| \leq \gamma_{V}(\rho), \\
& \left|\widetilde{V}_{N}(x)-\widetilde{V}_{N}\left(x^{e}\right)\right| \leq \gamma_{V}(\rho) .
\end{aligned}
$$

4. There are $N_{1} \in \mathbb{N}, \Delta \in \mathcal{L}$ such that

$$
J_{K}^{c l}\left(x, \mu_{N}(x)\right) \leq V_{N}(x)-V_{N}\left(x_{\mu_{N}}(K, x)\right)+\Delta(N)
$$

holds for all $x \in \mathbb{X}, N \geq N_{1}+1, K=1.6$

5. The function $\lambda$ from Assumption 3.1 is Lipschitz continuous on $\mathcal{B}_{\bar{\delta}}\left(x^{e}\right)$.

We check the five points above.

1. Strict dissipativity holds due to Assumption 3.1 boundedness of $\lambda$ on $\mathbb{X}$ follows from continuity of $\lambda$ and compactness of $\mathbb{X}$ which is Assumption 3.2 ,

\footnotetext{
${ }^{6}$ This is [8. Formula (18)] which in [8] is assumed to hold for all $K \in \mathbb{N}$. However, since
} here we only need 18 for $K=1$ it is sufficient to require 21 only for $K=1$. 
2. (a) Here, we can use [8, Theorem 5.3] (adapted to the non-averaged case), since $J_{N}\left(x, u_{N, x}^{\star}\right) \leq V_{N}(x) \leq N \ell\left(x^{e}, u^{e}\right)+\widetilde{V}_{N}(x)-\lambda(x)+$ $\lambda\left(x_{u_{N, x}^{\star}}(N)\right)$. Compactness of $\mathbb{X}$, continuity of $\lambda$ and the upper bound on $\widetilde{V}_{N}$ from Step 1 imply the existence of $C_{1}>0$ with $J_{N}\left(x, u_{N, x}^{\star}\right) \leq$ $N \ell\left(x^{e}, u^{e}\right)+C_{1}$. Hence, [8, Theorem 5.3] delivers the desired estimate with $C^{\prime}=C_{1}+\max _{x \in \mathbb{X}} 2|\lambda(x)|$.

(b) Proceeding analogously as in [8, Theorem 5.3] and with the help of (12), the desired property holds for

$$
\tilde{C}^{\prime}=\max _{x \in \mathbb{X}} \alpha_{V}\left(\left\|x-x^{e}\right\|\right) .
$$

3. Estimate 190 has been shown to hold in [8, Theorem 6.4] under dissipativity, a local controllability condition and boundedness of the rotated stage costs. A closer look at the proof of the theorem reveals that the latter two conditions can be substituted by 10 , Assumption 3.3 and local Lipschitz continuity of $\lambda$.

Estimate 20 can be deduced the following way: By (12), for each $x \in \mathbb{X}$ and $N \in \mathbb{N}$ the inequalities

$$
\alpha_{\ell}\left(\left\|x-x^{e}\right\|\right) \leq \widetilde{V}_{N}(x) \leq \alpha_{V}\left(\left\|x-x^{e}\right\|\right)
$$

hold and we conclude $\widetilde{V}_{N}\left(x^{e}\right)=0$ and thus

$$
\left|\widetilde{V}_{N}(x)-\widetilde{V}_{N}\left(x^{e}\right)\right|=\widetilde{V}_{N}(x) \leq \alpha_{V}\left(\left\|x-x^{e}\right\|\right) .
$$

4. Inequality 21 for $K=1$ has been shown to hold for $\Delta(N)=\varepsilon(N-1)$ in [8, Theorem 4.2]. For the sake of completeness we check that the assumptions of Therorem 3.7 include those of [8, Theorem 4.2]: Condition (a) follows from continuity of $\ell$ and $f$, condition (b) is estimate 19 which we have shown to hold above. Condition (c) can be concluded as in 8, Theorem 5.6]. To this end, we conclude [8, Assumption 5.5] from Assumptions 3.23 .4 as follows: Similiar to Step 1 we construct an upper bound for $\widetilde{J}_{N}(x, u)$. This yields an upper bound for $J_{N}(x, u)$, too, since 
both functionals only differ by $\lambda(x), \lambda\left(x_{u}(N, x)\right)$ and $N \ell\left(x^{e}, u^{e}\right)$. Due to continuity of $\lambda$ and compactness of $\mathbb{X}$ the $\lambda$-terms can be bounded and we can proceed as in the proof of [8, Theorem 5.6].

5. This is Assumption 3.2 .

Step 3: exponential decay of $\delta$. In order to show that $\delta(N)$ in 13 converges to 0 exponentially fast we shall look at the construction of $\tilde{\delta}$ in $[8$, Theorem 7.6], cf. the derivation of (18). It holds that $\tilde{\delta}(N) \leq \varepsilon(N)+12 \gamma_{V}(\tilde{\varepsilon}(N))+L_{\lambda} \tilde{\varepsilon}(N)$ with $\varepsilon(N)$ from [8, Theorem 4.2], $\gamma_{V}$ from $(19), \tilde{\varepsilon} \in \mathcal{L}$ and $L_{\lambda}$ the Lipschitz constant of $\lambda$.

Exponential convergence of $\varepsilon(N)$ holds due to [5, Theorem 6.5] if the functions $\gamma_{V}$ in 219, (20) and $\gamma_{\ell}, \gamma_{f}$ in [8, Theorem 4.2] are polynomial. This holds for $\gamma_{\ell}$ and $\gamma_{f}$ due to the Assumptions 3.2, 3.3 and 3.5. Inspection of the proofs of (19) and 20 in Step 2 of this proof reveals that $\gamma_{V}$ is polynomial if $\ell$ satisfies Assumption 3.5. This yields exponential convergence of $\varepsilon(N)$. To prove the assertion it is thus sufficient to show that also $\tilde{\varepsilon}(N)$ can be chosen to converge to 0 exponentially fast.

In the proof of $\left[8\right.$, Theorem 7.6], $\tilde{\varepsilon}(N)$ must be chosen such that $Q_{\tilde{\varepsilon}(N)} \geq c N$ holds for some $c \in(7 / 8,1)$ for $Q_{\tilde{\varepsilon}(N)}$ from Step 2. In [5, Theorem 6.5] is was proven that the exponential turnpike property holds under the Assumptions of Theorem 3.7 including Assumption 3.5. More precisely, for each $P \in \mathbb{N}$ it was shown that for $\bar{\varepsilon}_{P}(N)=\tilde{K} \eta^{(N-P) /(2 p)}, \tilde{K}>0, \eta \in(0,1), p>0$, the inequality $Q_{\tilde{\varepsilon}_{P}(N)} \geq P$ holds. We claim that $\tilde{\varepsilon}(N):=\bar{\varepsilon}_{\lceil c N\rceil}(N)$ satisfies the desired properties, where $\lceil c N\rceil$ denotes the smallest integer $\geq c N$ : on the one hand, we have $\tilde{\varepsilon}(N)=\tilde{K} \eta^{(N-\lceil c N\rceil) /(2 p)} \leq \tilde{K} \eta^{1 /(2 p)} \eta^{(1-c) N /(2 p)}$, implying that $\tilde{\varepsilon}$ indeed decays exponentially. On the other hand, $Q_{\tilde{\varepsilon}_{P}(N)} \geq P$ directly implies the desired inequality $Q_{\tilde{\varepsilon}(N)} \geq\lceil c N\rceil \geq c N$

Proof of Theorem 3.11; " $\Leftarrow$ ": We first show the implication "Assumptions 3.9 and 3.10 and $(A, B)$ stabilizable $\Rightarrow$ practical asymptotic stability on each compact subset $S \subset \mathbb{R}^{n}$ " via the existence of a practical Lyapunov function. 
We proceed as in the proof of Theorem 3.7

Step 1: proof of [12]. According to [5, Proposition 4.3] the affine linear quadratic problem is strictly dissipative with storage function $\lambda(x)=\nu^{T} x$ and $\alpha_{\ell}(r)=C_{1} r^{2}$ for some vector $\nu \in \mathbb{R}^{n}$ and some constant $C_{1}>0$. This implies the lower bound in 12 . The upper bound can be concluded as follows. In the proof of [5. Proposition 4.3] it was shown, that the rotated stage costs are of the form

$$
\tilde{\ell}(x, u)=\left(x-x^{e}\right)^{T} R\left(x-x^{e}\right)+\left(u-u^{e}\right)^{T} Q\left(u-u^{e}\right),
$$

hence there is $C_{2}>0$ such that $\tilde{\ell}(x, u) \leq C_{2}\left(\left\|x-x^{e}\right\|^{2}+\left\|u-u^{e}\right\|^{2}\right)$. Since $(A, B)$ is stabilizable, for each $x \in \mathbb{R}^{n}$ there exists a control sequence $u$ of infinite length and constants $C_{3}>0, \sigma \in(0,1)$ independent of $x$, such that

$$
\left\|x_{u}(k, x)-x^{e}\right\| \leq C_{3} \sigma^{k}\left\|x-x^{e}\right\|, \quad\left\|u(k)-u^{e}\right\| \leq C_{3} \sigma^{k}\left\|x-x^{e}\right\|
$$

holds for all $k \geq 0$. Combining all estimates implies

$$
\tilde{\ell}\left(x_{u}(k, x), u(k)\right) \leq 2 C_{2} C_{3}^{2} \sigma^{2 k}\left\|x-x^{e}\right\|^{2} .
$$

We obtain

$$
\widetilde{V}_{N}(x) \leq \sum_{k=0}^{\infty} \tilde{\ell}\left(x_{u}(k, x), u(k)\right) \leq 2 C_{2} C_{3}^{2} /\left(1-\sigma^{2}\right)\left\|x-x^{e}\right\|^{2}=: \alpha_{V}\left(\left\|x-x^{e}\right\|\right) .
$$

Step 2: proof of 13) We show that the assumptions of Theorem 3.11 include those of $\left[8\right.$, Theorem 7.6] on any compact subset $S$ of $\mathbb{R}^{n}$. To this end, we check the five properties listed in the proof of Step 2 of Theorem 3.7.

1. According to [5, Proposition 4.3] the affine linear quadratic problem is strictly dissipative with storage function $\lambda(x)=\nu^{T} x$ and $\alpha_{\ell}(r)=C_{1} r^{2}$ for some vector $\nu \in \mathbb{R}^{n}$ and some constant $C_{1}>0$. This structure of the storage function yields boundedness of $\lambda$ on $S$.

2. Both estimates, (a) and (b), can be concluded as in the previous proof as we restrict the initial state to the compact set $S$. 
3. In order to obtain $(19)$ we have a closer look at the optimal value function $V_{N}(x)$. We first remark, that we can eliminate the additive constant $c$ in the system dynamics through a coordinate transformation. This does not change the structure of the stage costs, and, without loss of generality, we can assume that the system is given by dynamics $x(k+1)=A x(k)+B u(k)$ and stage costs $\ell(x, u)=x^{T} Q x+u^{T} R u+s^{T} x+v^{T} u$ with $R, Q$ symmetric and positive definite. It follows from the dynamic programming principle that for each $N \in \mathbb{N}$ the optimal value function has the form

$$
V_{N}(x)=x^{T} P_{N} x+b_{N}^{T} x+d_{N}
$$

with $P_{N}$ symmetric and positive definite. We remark, that the proof is completely analogue (but computationally more complex) to the standard linear quadratic case (cf. 2, Section 2.4] for the LQR) and that $P_{N}$ is the solution of the backward Riccati iteration for the LQR.

As shown in the proof of [5, Theorem 6.2], $V_{N}$ is bounded uniformly in $N$ on the compact set $S$. This yields existence of constants $C_{S}, D_{S}$ such that

$$
C_{S} \leq V_{N}(x) \leq D_{S}
$$

holds for all $x \in S$ and all $N \in \mathbb{N}$. This yields boundedness of the vector $d_{N}$. Now consider sequences $\left(x_{i}\right)_{i \in \mathbb{N}}$ in $S$ and $\left(N_{i}\right)_{i \in \mathbb{N}}$ in $\mathbb{N}$ with $x_{i}^{T} P_{N_{i}} x_{i} \rightarrow \infty$. By 25 this is only possible if $b_{N_{i}}^{T} x_{i} \rightarrow-\infty$. Then, $\left(-x_{i}\right)^{T} P_{N_{i}}\left(-x_{i}\right) \rightarrow \infty$ and $b_{N_{i}}^{T}\left(-x_{i}\right) \rightarrow \infty$, too, which contradicts 25. Hence, there is $K>0$ independent of $N$ such that $0 \leq x^{T} P_{N} x \leq K\|x\|^{2}$ for all $x \in S$, and with the same argument there is $M>0$ independent of $N$ such that $\left|b_{N}^{T} x\right| \leq M\|x\|$ on $S$.

The bounds on $b_{N}^{T} x$ immediately imply that the entries of $b_{N}$ are bounded on $S$. Since $P_{N}$ is symmetric and positive definite its spectral norm is given by $]^{7}\left\|P_{N}\right\|_{2}=\max _{\|x\|=1} x^{T} P_{N} x \leq \max _{\|x\|=1} K\|x\|^{2}=K$. Therefore, the entries of

\footnotetext{
${ }^{7}$ As in the proof of [13, Lemma 8.2.1].
} 
$P_{N}$ are bounded on $S$. Now, it follows from the uniformity of the deduced bounds that for all $N \in \mathbb{N}, x \in S$ it holds

$$
\begin{aligned}
\left|V_{N}(x)-V_{N}\left(x^{e}\right)\right| & \leq\left|x^{T} P_{N} x-\left(x^{e}\right)^{T} P_{N} x^{e}\right|+\left|b_{N}^{T}\left(x-x^{e}\right)\right| \\
& \leq K\left|\|x\|^{2}-\left\|x^{e}\right\|^{2}\right|+M\left\|x-x^{e}\right\| \\
& =K\left|\left(\|x\|+\left\|x^{e}\right\|\right)\left(\|x\|-\left\|x^{e}\right\|\right)\right|+M\left\|x-x^{e}\right\| \\
& \leq 2 K \max \{\|x\|: x \in S\}\left|\|x\|-\left\|x^{e}\right\|\right|+M\left\|x-x^{e}\right\| \\
& \leq C\left\|x-x^{e}\right\|, \quad C>0 .
\end{aligned}
$$

This concludes the proof of (19).

Inequality 20 can be concluded as in the proof of Theorem 3.7 .

4. Again, for this property we use [8, Theorem 4.2] whose conditions are fulfilled.

5. Since $\lambda$ is a linear function (cf. Step 1 of this proof) it is Lipschitz continuous on every neighborhood of the equilibrium.

Step 3: exponential decay of $\delta$. Completely analogous to Step 3 of the proof of Theorem 3.7 using [5, Theorem 6.2] instead of [5, Theorem 6.5].

"६": Let the closed loop system be practically asymptotically stable on some compact subset $S \subset \mathbb{R}^{n}$ with $\varepsilon \rightarrow 0$ as $N \rightarrow \infty$. Then, for each $x \in S$ we can choose $N$ large enough such that the feedback steers the closed loop into an arbitrarily small neighborhood of $x^{e}$. This implies stabilizability of $(A, B)$.

\section{References}

[1] R. Amrit, J. B. Rawlings, D. Angeli, Economic optimization using model predictive control with a terminal cost, Annual Rev. Control 35 (2011) 178-186.

[2] B. D. Anderson, J. B. Moore, Optimal control: linear quadratic methods, vol. 1, Prentice Hall Englewood Cliffs, NJ, 1990. 
[3] D. Angeli, R. Amrit, J. B. Rawlings, On average performance and stability of economic model predictive control, IEEE Trans. Autom. Control 57 (7) (2012) 1615-1626.

[4] W. A. Brock, L. Mirman, Optimal economic growth and uncertainty: the discounted case, J. Econ. Theory 4 (1972) 479-513.

[5] T. Damm, L. Grüne, M. Stieler, K. Worthmann, An exponential turnpike theorem for dissipative discrete time optimal control problems, SIAM J. Control Optim.To appear.

[6] M. Diehl, R. Amrit, J. B. Rawlings, A Lyapunov function for economic optimizing model predictive control, IEEE Trans. Autom. Control 56 (2011) 703-707.

[7] L. Fagiano, A. R. Teel, Generalized terminal state constraints for model predictive control, Automatica 49 (2013) 2622-2631.

[8] L. Grüne, Economic receding horizon control without terminal constraints, Automatica 49 (3) (2013) $725-734$.

[9] L. Grüne, J. Pannek, Nonlinear Model Predictive Control. Theory and Algorithms, Springer-Verlag, London, 2011.

[10] M. Heidarinejad, J. Liu, P. D. Christofides, Economic model predictive control of nonlinear process systems using Lyapunov techniques, AIChE Journal 58 (2012) 855-870.

[11] M. A. Müller, F. Allgöwer, Robustness of steady-state optimality in economic model predictive control, in: Proceedings of the 51st IEEE Conference on Decision and Control - CDC2012, Maui, Hawaii, 2012, pp. 1011-1016.

[12] A. Porretta, E. Zuazua, Long time versus steady state optimal control, SIAM J. Control Optim. 51 (2013) 4242-4273. 
[13] E. D. Sontag, Mathematical Control Theory, 2nd ed., Springer Verlag, New York, 1998. 